\title{
Hautkrankheiten - von scheinbar harmlos bis lebensbedrohlich
}

$\mathrm{D}$ ie dermatologische Tätigkeit, so ein weit verbreiteter Irrglaube, ist deshalb so attraktiv, weil Patienten niemals versterben und aufgrund ihrer schleichenden chronischen Hauterkrankungen immer wieder die Praxis des Hautarztes aufsuchen. Dieses Vorurteil wird der Vielfältigkeit und Komplexität der Dermatologie in keinster Weise gerecht. Hauterkrankungen können schwere lebensbedrohliche Zustände darstellen, die eine sofortige Intervention erfordern. Zu den häufigsten gefährlichen und lebensbedrohlichen Situationen im dermatologischen Alltag zählen sicher die allergischen Reaktionen. Die Zunahme von Bienen- und Wespengiftallergien und der daraus resultierende Anstieg der anaphylaktischen Reaktionen hat in unserer Klinik dazu geführt, eine dermatologische „Überwachungseinheit" mit hochmodernem Intensivequipment zu etablieren. Bei solchen Patienten ist eine Hyposensibilisierung in der Anfangsphase stationär unter intensivmedizinischen Kautelen indiziert. Der steigende Medikamentenkonsum sowie die ständig neu auf den Markt kommenden Medikamente haben zu einer dramatischen Zunahme an Arzneimittelexanthemen in den letzten Jahren geführt. Dabei reicht die Bandbreite der Hautveränderungen von diskreten, makulo-papulösen Exanthemen über das Erythema exsudativum multiforme mit Schleimhautaffektion bis zur Maximalvariante der Arzneimittelnebenwirkung, der toxisch-epidermalen Nekrolyse. Solche Notfallsituationen machen selbstverständlich ein rasches ärztliches Eingreifen unabdingbar. Häufig sind weiterhin allergische Reaktionen durch Nahrungsmittel. Auch hier reicht das Spektrum klinischer Veränderungen von der diskreten Urtikaria über Angioödeme bis zum anaphylaktischen Schock.

Herpesinfektionen sind häufig und meist ambulant gut beherrschbar. Dies gilt auch für den unkomplizierten Herpes zoster. Der Herpes zoster im Kopfund Gesichtsbereich ist - insbesondere bei Patienten mit Immundefizienz - eine Indikation zur stationären Behandlung. Die Vermeidung schwerer Zosterneuralgien ist hier nur durch frühzeitige adäquate antivirale und analgetische Therapie möglich. Eine weitere Indikationen zur schnellen sofortigen Therapie stellt das Eczema herpeticatum, eine massive HerpesInfektion beim atopischen Ekzem, dar. Auch bei bakteriellen Infektionen der Haut gilt es, Frühzeichen zu erkennen und schnell suffizient zu therapieren. Das nekrotisierend verlaufende Erysipel, ein potentiell lebensbedrohliches Krankheitsbild beim multimorbiden, prädisponierten Patienten, kann durch eine frühe suffiziente Antibiose in Kombination mit zeitgerechter Nekrosektomie erfolgreich behandelt werden.

Die Erythrodermie, unabhängig von der zugrundeliegenden Erkrankung, stellt durch mögliche Wärme- und Flüssigkeitsverluste per se einen potentiell lebensbedrohlichen Zustand dar. Die adäquate System- und Lokaltherapie ist meist nur unter stationären Bedingungen realisierbar. Das hereditäre Angioödem, eine durch einen genetischen Defekt des C1-Esterase-Inhibitors bedingte Erkrankung, ist zwar sehr selten, jedoch, wenn das entsprechende Protein am Anfang nicht sofort verabreicht wird, ein lebensbedrohliches Krankheitsbild. Hochdosierte Glukokortikosteroide und Antihistaminika sind wirkungslos. Der Verbrennungspatient erfordert eine interdisziplinäre Zusammenarbeit. Verbrennungen von über 15\% der Körperoberfläche bei Erwachsenen sowie schwere dermale Verbrennungen mit Indikation zu plastisch-chirurgischen Maßnahmen müssen in einem Verbrennungszentrum behandelt werden.

Mögen die folgenden Kurzübersichten über Arzneimittelexantheme, Herpes Zoster und atopisches Ekzem verdeutlichen, dass Übergänge zwischen scheinbar harmlosen Hautveränderungen und lebensbedrohlichen Krankheitsbildern fließend sein können und frühzeitige diagnostische und therapeutische Intervention erfordern.
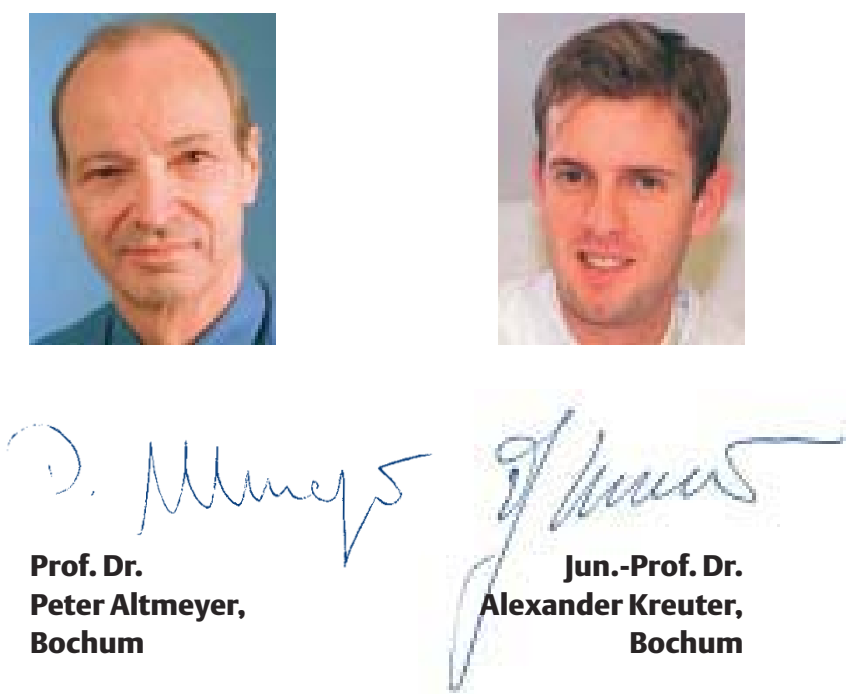